Annuaire suisse de politique de développement

15 | 1996

Annuaire Suisse - Tiers Monde 1996

\title{
Le système de commerce multilatéral et la question des normes du travail
}

\section{Didier Chambovey}

\section{(2) OpenEdition}

1 Journals

Édition électronique

URL : http://journals.openedition.org/aspd/1517

DOI : $10.4000 /$ aspd. 1517

ISSN : 1663-9669

Éditeur

Institut de hautes études internationales et du développement

\section{Édition imprimée}

Date de publication : 1 mars 1996

Pagination : 189-214

ISSN : 1660-5934

\section{Référence électronique}

Didier Chambovey, «Le système de commerce multilatéral et la question des normes du travail »,

Annuaire suisse de politique de développement [En ligne], 15 | 1996, mis en ligne le 16 mai 2013, consulté le 08 septembre 2020. URL : http://journals.openedition.org/aspd/1517 ; DOI : https:// doi.org/10.4000/aspd. 1517 


\title{
Le système de commerce multilatéral et la question des normes du travail
}

\author{
Didier Chambovey ${ }^{1}$
}

\section{Introduction}

Les interactions des normes du travail et du commerce s'inscrivent aujourd'hui dans le contexte d'une intégration croissante des échanges internationaux de marchandises et de services, d'information et de technologie ainsi que des mouvements de capitaux. De même, le processus de production tend à se mondialiser par le biais d'investissements directs, de prises de participation et d'alliances stratégiques. Ce phénomène de globalisation de l'économie s'est renforcé sous l'effet des progrès techniques des transports et des télécommunications qui réduisent considérablement les coûts de transaction entre les différentes régions de la planète. II a également été facilité par les politiques d'ouverture aux échanges pratiquées depuis la seconde guerre mondiale dans les pays occidentaux et plus récemment par de nombreux pays en développement ou en transition vers une économie de marché. De la sorte, les possibilités de désagréger les différents stades de la fabrication d'un produit ou de la prestation d'un service et de les localiser dans les pays qui offrent, pour chacun d'eux, les conditions de production ou de commercialisation les plus avantageuses se sont notablement accrues ${ }^{2}$. Ces mutations ont favorisé l'essor de firmes

1. Les opinions exprimées dans cet article sont personnelles et n'engagent que l'auteur.

2. Il en est résulté une intensification des échanges transfrontières intra-firmes ou intrabranches de produits semi-finis issus d'unités de production hautement spécialisées. Ce phénomène a été analysé par Henri Gétaz, Les échanges intra-industriels. Analyse théorique et empirique pour la Suisse. Thèse présentée à l'École des Hautes Études Commerciales de l'Université de Lausanne, 1994. 
multinationales qui ont progressivement perdu leur base nationale et qui conçoivent leur stratégie à l'échelle du monde. La globalisation des marchés et de la production présente certes de vastes possibilités de développement économique et d'expansion de l'emploi aux pays qui participent à l'échange international. Elle a néanmoins pour corollaires une profonde transformation de la division internationale du travail et la nécessité, pour les économies nationales, de faire preuve d'une capacité d'adaptation et d'innovation permanente.

Dans un contexte de globalisation, les facteurs de production sont de plus en plus mobiles si bien que le capital et la technologie, qui s'est déjà largement uniformisée dans les pays industrialisés, se déplacent aussi vers les pays en développement où les coûts salariaux sont moins élevés. Les effets de cette évolution sur la division internationale du travail peuvent être analysés à l'aide d'un modèle d'équilibre général du type Heckscher-Ohlin-Samuelson ${ }^{3}$. Ce modèle met en évidence la relation entre la dotation factorielle et les avantages comparatifs: les pays tendent à exporter les biens dont la production requiert un usage relativement intensif des facteurs de production qu'ils possèdent en quantités relativement abondantes. En raison de l'uniformisation croissante du capital et de la technologie, la spécialisation de la production est de plus en plus fonction du niveau de qualification de la main-d'oeuvre. Aussi, les pays en développement dotés d'une abondante main-d'oeuvre peu qualifiée et bon marché $e^{4}$, bénéficient indéniablement d'un avantage comparatif dans les productions intensives en travail de faible qualification (par exemple des segments importants des secteurs de l'électronique grand public, de la chaussure, du textile et de l'habillement). De plus, le surcoût des salariés peu qualifiés des pays industrialisés ne peut plus être compensé par une meilleure dotation en capital et en technologie. Dans une économie globalisée, les travailleurs peu qualifiés des pays en développement engagés dans les secteurs qui participent aux échanges internationaux se trouvent pratiquement sur le même marché de l'emploi que leurs homologues des pays industrialisés. Dans ces derniers pays ${ }^{5}$, le taux

3. Le lecteur intéressé aux développements récents de la théorie de l'échange international se reportera à l'article de J. Hellier, “La similitude dans l'échange international: une revue critique des approches théoriques", Problèmes économiques, $\mathrm{n}^{0} 2.348$, 1993. Une application du modèle Heckscher-Ohlin-Samuelson à la question des échanges et des normes du travail a été proposée par Brown D. K., Deardorf A. V. et Stern R. M., "International Labour Standards and Trade: A Theoretical Analysis", communication présentée à la réunion du Projet Fairness Claims and Gains from Trade, University of Minnesota Law School, 29-31 juillet 1993.

4. Eu égard à l'évolution démographique, cette caractéristique est appelée à se renforcer dans les pays en développement. Selon les projections, environ $99 \%$ du milliard de travailleurs qui entreront sur le marché du travail dans les 30 prochaines années seront des ressortissants d'économies à revenu faible ou intermédiaire (cf. The World Bank, World Development Report 1995. Workers in an Integrating World, Oxford University Press, New York, 1995, 7).

5. Aux États-Unis, le salaire réel des hommes avec une formation inférieure aux études secondaires a diminué de $23 \%$ entre 1972 et 1994. 
d'emploi des travailleurs peu qualifiés par rapport à celui des travailleurs très qualifiés a diminué et les salaires réels perçus par la main-d'oeuvre faiblement qualifiée ont peu augmenté, voire régressé, depuis les années 1970. En revanche, les pays industrialisés sont d'importants producteurs et exportateurs de produits de haute technologie et le nombre d'emplois occupés par un personnel qualifié y a augmenté.

Les gouvernements des économies intégrées dans l'échange international admettent que freiner ou inverser le cours de la globalisation au moyen de mesures protectionnistes irait à l'encontre de leurs intérêts ${ }^{6}$. Conscients des ajustements structurels que cela suppose, ils ont au contraire affirmé leur voIonté de tirer parti des retombées positives du processus de globalisation et ont intensifié leur coopération économique, tant sur le plan régional que multilatéral, dans le but d'établir des conditions-cadre qui facilitent la mobilité transfrontière des biens, des services et des facteurs de production. Ils cherchent ainsi à créer un environnement réglementaire propice à la croissance économique et à la reprise de l'emploi dans l'ensemble de la communauté internationale. Ces initiatives se sont traduites par une plus grande convergence des politiques économiques nationales. Elles n'ont toutefois pas été accompagnées, à l'échelle de la planète, d'un rapprochement comparable des législations sociales dont certaines, telles celles relatives aux normes du travail, pourraient avoir une influence sur les conditions de concurrence. Dans un contexte de mondialisation du marché de l'emploi, des normes du travail médiocres sont parfois perçues comme une forme de concurrence déloyale à l'origine de pertes d'emplois dans les pays qui maintiennent des normes plus sévères. Certains acteurs du champ économique soutiennent qu'en l'absence du respect universel de normes fondamentales du travail la pression de la concurrence déclenche une course à la dévaluation sociale dont pâtissent aussi bien les pays industrialisés que les pays en développement. Pour enrayer cette spirale descendante, ils proposent d'intégrer des normes du travail minimales dans la politique commerciale internationale ${ }^{7}$. Cette proposition est cependant loin d'emporter l'adhésion de nombreux pays (en particulier des pays en développe-

6. Cf. OCDE, Réunion du Conseil de l'OCDE au niveau ministériel, Communiqués de presse du 8 juin 1994 et du 24 mai 1995, Division de la presse de l'OCDE, Paris.

7. Cf. International Confederation of Free Trade Union, International Workers' Rights and Trade: The Need for Dialogue, September 1994; Confédération des syndicats chrétiens de Suisse, Déclaration de Berne, Pain pour le Prochain; Déclaration sur la clause sociale des syndicats, organisations de développement, de protection de l'environnement, de femmes, de défense des droits de l'homme, 8 février 1995. Déclaration de Berne, "Made in Dignity: Dossier clause sociale», Vers un développement solidaire, no 127, février 1995, 3-11. Dans une résolution adoptée le 9 février 1994 par 190 voix contre 29 le Parlement européen s'est explicitement prononcé en faveur de l'introduction d'une "clause sociale" dans le système multilatéral du commerce mondial. 
ment), associations professionnelles et autres analystes ${ }^{8}$. Ceux-ci considèrent que l'instauration d'un tel dispositif ne trouve aucune justification dans l'analyse économique et qu'elle ouvrirait la voie à des mesures protectionnistes dont les pays du Sud auraient à souffrir.

Cette controverse n'a pas laissé indifférents les participants au système commercial multilatéral. Aussi, à l'instigation des Etats-Unis ${ }^{9}$ et de la France, le débat sur la dimension sociale du commerce international a-t-il été relancé lors de la conclusion du cycle d'Uruguay à Marrakech (15 avril 1994). Un certain nombre de délégations, ont demandé que la relation entre le système commercial multilatéral et les normes du travail internationalement reconnues soit examinée en vue de son inclusion comme point additionnel dans le programme de travail de l'Organisation mondiale du commerce (OMC). Par ailleurs, en juin 1994, la conférence ministérielle de l'OCDE a décidé d'étudier la relation entre les échanges, l'emploi et les normes du travail internationalement reconnues. L'objet de ces travaux est de déterminer s'il existe effectivement un lien entre la compétitivité internationale d'un pays et les normes du travail qu'il applique. Ils devraient aboutir à la présentation d'un rapport en 1996. Ce document complétera les analyses déjà effectuées par l'OCDE ${ }^{10}$. En outre, une réflexion sur la dimension sociale du commerce international a été engagée lors de la 81ème . session de la Conférence annuelle de l'Organisation internationale du travail (juin 1994). Cette dernière a également publié un rapport consacré au thème de la globalisation et de l'emploi'1.

L'objet de cet article n'est pas apologétique. Nous nous proposons plutôt de dresser un panorama des propositions, des actions concrètes et des recherches afférentes à la question des interactions du commerce et des normes du travail. Nous évoquerons également le rôle dévolu aux diverses organisations internationales concernées par cette problématique et les conditions à réunir pour engager des travaux susceptibles d'aboutir à des solutions mutuellement acceptables au sein de l'OMC. A ce jour, les connaissances scientifiques dans ce domaine sont lacunaires, plus particulièrement en ce qui concerne les vérifi-

8. Cf. Bhagwati J., "Free Trade: Old and New Challenges", The Economic Journal, vol. 104, no 423, March 1994, 231-246; Kohr M., "Et la clause sociale ?", Courrier de la planète, $n^{\circ} 22$, avril-mai 1994; Mahathir M., "East Asia will find its own Roads to Democracy", International Herald Tribune, 17 May 1994, 6.

9. Avec l'avènement d'un Congrès dominé par les Républicains, on ne peut exclure un retournement de la politique américaine en la matière. Les députés républicains se sont en effet déclarés hostiles à l'utilisation de mesures commerciales pour atteindre des objectifs relatifs aux normes du travail (Cf. Economic Strategy Institute, Working Together. A Bipartisan Trade Agenda, Washington, D.C., January 1995, 19-21).

10. Cf. OCDE, L'étude de l'OCDE sur l'emploi. Faits. Analyses. Stratégies, OCDE, Paris, 1994; OCDE, Perspectives de l'emploi, OCDE, Paris, 1994; OCDE, Échanges et normes du travail. Examen des principales questions, OCDE, Paris, 1995.

11. Bureau international du travail, L'emploi dans le monde. Un rapport du BIT, Genève, 1995. 
cations empiriques. Les travaux en cours permettront certainement d'améliorer notre compréhension du sujet sans pour autant apporter des réponses définitives et univoques aux nombreuses questions posées dans le débat public. Les chercheurs sont en effet confrontés à la difficulté méthodologique qui consiste à isoler la part revenant à une variable qualitative que l'on ne peut appréhender statistiquement (les normes du travail) dans l'explication de variables économiques telles que les performances à l'exportation ou les coûts salariaux. Les études actuellement disponibles permettent toutefois de tirer certains enseignements utiles dont nous ferons état. Notre intention n'est pas de nous prononcer sur l'opportunité morale du respect universel de certaines normes du travail, mais de contribuer à l'éclaircissement de la relation entre ces normes et le phénomène de globalisation des économies.

\section{Les normes du travail dans les accords commerciaux internationaux}

\subsection{Brève rétrospective}

La proposition de lier la libéralisation des échanges au respect de normes du travail spécifiques n'est pas nouvelle. Elle s'est concrétisée par l'introduction de "clauses sociales" dans de nombreux accords commerciaux ou législations, qu'il s'agisse d'actions unilatérales décidées par un ou plusieurs gouvernements, ou du résultat d'une négociation plurilatérale entre Etats désireux d'améliorer les conditions de travail sur le territoire des parties contractantes. De manière générale, ces entreprises ont pour objet d'établir des règles du jeu uniformes, recouvrant également la dimension sociale du commerce international, afin d'éviter, dans un contexte d'ouverture aux échanges, que des réglementations divergentes ne confèrent un avantage comparatif aux pays où les normes du travail sont moins rigoureuses. Procéder à une recension exhaustive des initiatives prises en la matière confine pratiquement à la gageure. Nous nous limiterons donc à mentionner celles qui revêtent un intérêt particulier dans le cadre de cet article.

En 1881 déjà, la Federation of Organized Trades and Labour ${ }^{12}$ a fait pression sur le Congrès américain pour obtenir des moyens de protéger les producteurs américains de la concurrence des pays à bas salaires. La protection exigée devait prendre la forme d'un droit de douane compensateur imposé sur les produits en provenance de ces pays. Ce principe a été ancré dans les Tariff Acts de 1922 et $1930^{13}$. Sur le plan multilatéral, il convient de signaler l'Organisation internationale du commerce (OIC) qui aurait dû constituer, en complé-

12. A laquelle l'American Federation of Labour a succédé.

13. Cf. Charnovitz S., "The influence of international labour standards on the World Trading Regime. A Historical Overview", International Labour Review, vol. 126, 1987, 565-584. 
ment des institutions de Bretton Woods, le troisième pilier de l'ordre économique mondial de l'après-guerre. Ses statuts, consignés dans la Charte de $\mathrm{La}$ Havane, comprenaient une disposition (article 7) relative aux normes du travail par laquelle les Etats Membres reconnaissaient

“1. que les mesures relatives à l'emploi doivent pleinement tenir compte des droits qui sont reconnus aux travailleurs par des déclarations, des conventions et des accords intergouvernementaux ... Les Etats Membres reconnaissent que l'existence de conditions de travail non équitables, particulièrement dans les secteurs de la production travaillant pour l'exportation, crée des difficultés aux échanges internationaux. En conséquence, chaque Etat Membre prendra toutes les mesures appropriées et pratiquement réalisables en vue de faire disparaître ces conditions sur son territoire ... Pour toutes les questions relatives aux normes de travail qui pourraient lui être soumises conformément aux dispositions de l'article 94 ou de l'article $95^{14}$, l'Organisation consultera l'Organisation internationale du travail et collaborera avec elle».

La Charte de l'OIC a certes été acceptée lors d'une Conférence des Nations Unies sur le commerce et l'emploi à La Havane en 1948 mais elle n'est jamais entrée en vigueur. En effet, le gouvernement des Etats-Unis décida, en 1950, de ne pas la soumettre au Congrès pour ratification. L'OIC ne vit jamais le jour. Seules les règles commerciales contenues dans le projet de Charte, entrées provisoirement en vigueur le 1er janvier 1948, subsistèrent sous le nom d'Accord général sur les tarifs douaniers et le commerce (GATT). A l'exception d'une disposition que nous évoquerons dans la section 2.3, le GATT ne reprenait aucun principe relatif aux normes du travail. C'est d'ailleurs sans succès que les Etats-Unis s'employèrent, en 1953, à introduire l'article 7 de la Charte de la Havane dans le GATT. Ils sont revenus à la charge en 1986 en proposant de faire figurer la question des droits des travailleurs au programme de négociation du cycle d'Uruguay. Ce postulat s'est heurté à une vive opposition des pays en développement et n'a pas été retenu.

Des articles ayant trait aux conditions de travail ont également été introduits dans les accords sur les produits de base suivants: Accords sur l'étain (1954), sur le sucre (1968), sur le cacao (1975) et sur le caoutchouc naturel (1979). Toutefois, ces dispositions s'apparentent à des déclarations d'intention et ne sont pas assorties de mécanismes de contrôle et de sanction ${ }^{15}$. La Convention de Lomé de 1984 conclue entre l'Union européenne et les Etats ACP (Asie, Caraïbes, Pacifique) contient également des principes généraux relatifs au respect de normes du travail.

14. Ces articles correspondent à l'article XXIII de l'Accord général sur les tarifs douaniers et le commerce concernant le règlement des différends.

15. Cf. Kullmann U., "Fair Labour Standards" in International Commodity Agreements", Journal of World Trade Law, 1980, 527-535. 
Les propositions ou instruments énumérés ci-dessus visant à rendre effective une "clause sociale" insérée dans des accords commerciaux se sont révélés inopérants, soit par défaut de volonté politique ou de force contraignante, soit en raison de difficultés pratiques relatives à leur application. Plus récemment, les Etats-Unis ont entrepris des démarches unilatérales visant à lier des programmes d'aide ou des initiatives commerciales à l'amélioration des normes du travail dans les pays en développement. II s'agit en particulier du Carribean Basin Economic Recovery Act (1983), du Système généralisé de préférences (1984), de l'Overseas Private Investment Corporation (1985) et de la Section 301 de l'Omnibus Trade and Competitiveness Act (1988) ${ }^{16}$. Le fonctionnement du Système généralisé de préférences américain revêt sans doute le plus grand intérêt dans ce contexte. Il en sera question dans la prochaine section.

De surcroît, pour donner suite aux préoccupations exprimées par de nombreux groupes à propos des conséquences négatives que l'Accord de libreéchange nord-américain (ALENA) pourrait avoir sur l'emploi, les salaires et les conditions de travail, les Etats-Unis ont amené leurs partenaires ${ }^{17}$ à négocier un accord complémentaire sur ces questions (1993): l'Accord nord-américain de coopération dans le domaine du travail ${ }^{18}$. En substance, cet instrument engage chaque partie à respecter sa propre législation du travail. La liste des cas qui peuvent être soumis à une procédure de règlement des différends ne couvre cependant que des domaines limités et bien définis. II s'agit des situations dans lesquelles il y a non-application systématique par un pays de ses propres normes du travail en matière de sécurité et de santé, de travail des enfants et de salaires minima. De plus, le cas en question doit affecter les échanges à l'intérieur de la zone de libre-échange. En cas d'échec final des tentatives de règlement à l'amiable, la procédure de règlement des différends peut conduire à l'imposition d'une amende, voire à des sanctions commerciales si cette amende n'est pas acquittée.

\subsection{Les Systèmes généralisés de préférences (SGP) des Etats-Unis et de l'Union européenne}

En 1984, un amendement au schéma américain du Système généralisé de préférences $^{19}$ lie l'octroi de ce régime au respect de normes du travail internationalement reconnues. On n'exige pas ici le respect intégral de ces

16. Cf. van Liemt G., “Minimum Labour Standards and International Trade: Would a Social Clause Work ?", International Labour Review, Vol. 128, 1989, 433-448.

17. Canada et Mexique.

18. The NAFTA. Supplement Agreements, U.S. Government Printing Office, Washington D.C., 1993.

19. Le Système généralisé de préférences (SGP) consiste à offrir aux pays en développement des avantages sous forme de réductions tarifaires pouvant aller jusqu'à l'exemption. Son utilisation est discrétionnaire et les préférences peuvent être reti- 
normes telles qu'elles sont définies dans les conventions de l'OIT mais que des mesures soient prises à cet effet. L'amendement se référait aux principes suivants:

- le droit d'organisation et de négociation collective;

- l'interdiction du travail forcé;

- un âge minimal d'admission à l'emploi des enfants;

- des conditions décentes d'emploi en matière de salaire minimal, de durée du travail et d'hygiène et de sécurité sur la place de travail.

Le programme SGP des Etats-Unis fait l'objet d'une procédure d'examen dans le cadre de laquelle un organe (The GSP Subcommittee) composé de représentants de l'administration fédérale statue sur les requêtes qui lui sont adressées. Ces examens peuvent également porter, selon la nature de la demande, sur l'observation des critères sociaux énoncés ci-dessus. A supposer qu'elles acceptent d'entrer en matière, les autorités peuvent décider de prolonger la période d'examen, de mettre un terme à ce dernier si le pays visé prend des mesures jugées appropriées ou de suspendre l'application du régime préférentiel si le pays visé ne se conforme pas aux conditions prévues dans la législation sur le SGP. Parallèlement aux examens, des négociations ont lieu en vue de trouver une solution acceptable pour les deux partenaires; les sanctions commerciales ne sont appliquées qu'en dernier recours. Les syndicats de travailleurs sont évidemment de grands utilisateurs du mécanisme d'examen qu'ils souhaiteraient rendre plus expéditif et plus sévère. Sur les 34 pays qui ont fait l'objet d'une demande d'examen au titre de la conditionnalité sociale entre 1984 et 1994, seuls sept pays ont perdu les bénéfices du SGP américain (Chili, Liberia, Mauritanie, Myanmar, République centrafricaine, Soudan et Syrie). Deux d'entre eux (Chili et République centrafricaine) ont été réadmis depuis. L'efficacité du mécanisme en tant que moyen d'améliorer durablement le droit du travail et son application dans les pays examinés est contestée. II a notamment été relevé que les avantages découlant des préférences tarifaires étaient insuffisants pour inciter les pays bénéficiaires à changer durablement leurs législations et pratiques nationales ${ }^{20}$.

On ne saurait dire à ce stade si l'expérience des Etats-Unis pourrait faire tache d'huile. A ce titre, il convient de relever que l'Union européenne a inclus une "clause sociale" dans son nouveau schéma de préférences tarifaires entré

rées sur la base de critères fixés par le pays qui octroie le traitement SGP. II s'agit là d'une exception à la clause de la nation la plus favorisée du GATT car les concessions tarifaires accordées aux pays en développement dans le cadre du SGP ne doivent pas être étendues aux pays développés. Cf. Traitement différencié et plus favorable, réciprocité et participation plus complète des pays en voie de développement (ou "Clause d'habilitation"), GATT Sup. n²6 IBDD 223.

20. Cf. United States General Accounting Office, International Trade. Assessment of the Generalized System of Preferences Program, GAO/GGD-95-9, November 1994, 9-11. 
en vigueur le 1er janvier $1995^{21}$. Pour partie, cette dernière sera de nature incitative et se traduira par l'octroi de réductions tarifaires additionnelles, venant s'ajouter à la marge préférentielle de base, aux pays bénéficiaires qui s'engageront à respecter les principes contenus dans des conventions de l'OIT relatives à la liberté syndicale, au droit de négociation collective et au travail des enfants. Ce régime spécial d'encouragement ne sera applicable qu'à partir du 1 er janvier 1998. D'autres dispositions entrées en vigueur le 1er janvier 1995 prévoient la possibilité de retirer partiellement ou totalement le régime de base en raison de pratiques telles que l'esclavage ou l'exportation de produits fabriqués dans les prisons. Ces précédents ouvrent évidemment la voie à une utilisation de plus en plus discrétionnaire du SGP et pourraient entraîner d'autres pays à en faire de même.

\subsection{Le système commercial multilatéral (GATT/OMC)}

On l'a vu, la "clause sociale" de la Charte de La Havane n'a pas été incorporée dans l'Accord général sur les tarifs douaniers et le commerce (GATT). Cet Accord qui fait aujourd'hui partie intégrante ${ }^{22}$ des accords multilatéraux sur le commerce des marchandises de l'OMC contient toutefois, au chapitre des exceptions générales, une référence expresse aux droits des travailleurs. II s'agit de l'article XX (e) qui, sans constituer une prohibition du travail carcéral, autorise les mesures contre le commerce des articles fabriqués dans les prisons. Cette disposition n'implique pas une action collective mais laisse à chaque Membre de l'OMC la discrétion de prendre des mesures commerciales selon son appréciation de la situation. A l'exception des circonstances prévues à l'article XX(e), l'importation d'un produit ne saurait être subordonnée au respect, par le pays exportateur, de conditions de production spécifiques relatives aux normes du travail.

Les instruments juridiques de l'OMC prévoient également des moyens de défense contre certaines formes de concurrence telles que le commerce des produits faisant l'objet d'un dumping ou bénéficiant d'une subvention. Des contre-mesures sous forme de droits antidumping ou compensateurs sont autorisées dans des conditions strictement définies; elles visent à neutraliser la marge de dumping calculée par rapport à une "valeur normale" ou à annuler l'effet de sous-enchère d'une subvention. En outre, le pays qui recourt à ces mesures doit établir que le dumping ou les subventions incriminés portent préjudice à une industrie nationale existante ou retarde l'établissement de cette industrie. Selon un fort courant de pensée, l'application de normes du travail

21. Cf. Règlement (CE) no 3281/94 du Conseil du 19 décembre 1994 portant application d'un schéma pluriannuel de préférences tarifaires généralisées pour la période 19951998 à certains produits industriels originaires de pays en développement, JO Législation (1994), no L/348/1.

22. Résultats des négociations commerciales multilatérales du cycle d'Uruguay. Textes juridiques, Secrétariat du GATT, Genève, 1994, 21. 
médiocres dans un pays exportateur constituerait une forme de concurrence déloyale au détriment de pays importateurs où ces normes seraient plus rigoureuses. Des notions telles que le "dumping social" ou le "subventionnement social" sont ainsi apparues de longue date dans le débat sur la "clause sociale». Peut-on, par analogie, inclure ces pratiques dans le champ des moyens de défense commerciale dont disposent les Membres de l'OMC?

Au sens de l'article 2 de l'Accord sur les mesures antidumping de l'OMC, un produit est considéré comme faisant l'objet d'un dumping si le prix à l'exportation de ce produit est inférieur au prix comparable pratiqué au cours d'opérations commerciales normales pour le produit similaire dans le pays exportateur. Lorsque cette méthode n'est pas applicable pour des raisons techniques, la marge de dumping peut être déterminée par comparaison du prix à l'exportation avec le coût de production dans le pays d'origine majoré d'un montant raisonnable pour les frais de commercialisation et pour les bénéfices. II s'agit ici d'opérations purement comptables exemptes de toutes considérations quant aux conditions dans lesquelles les produits ont été fabriqués. Ainsi, si le prix pratiqué dans le pays producteur n'est pas supérieur au prix à l'exportation, on ne conclura pas à l'existence d'un dumping quelle que soit la condition des travailleurs dans ce pays. De même, dans les cas où la marge de dumping est déterminée par rapport aux coûts de production, l'enquête devra se fonder sur les frais effectivement encourus par le producteur (sur la base de ses registres de comptabilité). II n'est pas possible de majorer ces coûts parce que la rémunération de la main-d'oeuvre serait jugée artificiellement basse en raison de normes du travail insuffisantes.

Au sens de l'article premier de l'Accord sur les subventions de l'OMC, une subvention est réputée exister s'il y a une contribution financière des pouvoirs publics ou s'il y a une forme quelconque de soutien des revenus ou des prix qui a directement ou indirectement pour effet d'accroître les exportations ou de réduire les importations d'un produit. A l'évidence, le refus d'accorder certains droits aux travailleurs ne constitue pas une contribution financière des pouvoirs publics. On pourrait néanmoins estimer qu'il en résulte un soutien des revenus dont l'effet indirect serait d'accroître les exportations ou de réduire les importations. II faudrait alors apporter la preuve que les normes du travail sont délibérément abaissées par une action gouvernementale à dessein de compresser les coûts de production de certaines entreprises ou branches de production. En outre, le montant de la subvention devrait être calculé en regard des conditions de travail normales qui prévaudraient en l'absence d'une telle intervention des autorités. L'opération confine pratiquement à la gageure. II serait donc extrêmement difficile de réunir les conditions nécessaires à l'imposition de droits compensateurs en respectant l'esprit de l'Accord sur les subventions.

En définitive, le système commercial multilatéral n'a pas été conçu pour veiller au respect universel de certaines normes du travail internationalement reconnues, d'autant plus que l'OMC ne connaît pas le mécanisme des sanctions collectives. On ne saurait pourtant affirmer qu'il se limite aux questions qui sont intrinsèquement commerciales. La libéralisation des échanges a progressi- 
vement porté sur des sujets touchant au commerce qui relevaient de la politique autonome des Etats, comme la protection des droits de propriété intellectuelle. L'Accord sur la propriété intellectuelle de l'OMC dispose en effet que les Membres feront en sorte que leur législation assure le respect de normes internationales de protection de la propriété intellectuelle et prévoient des mesures coercitives destinées à prévenir toute violation. La protection de la propriété intellectuelle bénéficie désormais du même degré de force contraignante que les dispositions des autres accords de l'OMC: un Membre qui manquerait à ses obligations s'exposerait ainsi à des sanctions commerciales autorisées dans le cadre de la procédure de règlement des différends ${ }^{23}$. Cet exemple explique l'attrait qu'exerce le système commercial multilatéral sur les groupes et les gouvernements qui désirent y inclure une "clause sociale". Pareille entreprise nécessiterait toutefois d'apporter des amendements substantiels aux accords de l'OMC et modifierait l'équilibre des droits et obligations négocié lors du cycle d'Uruguay.

A l'occasion de la conférence de Marrakech, il n'a pas été formellement convenu de faire figurer à l'ordre du jour de l'OMC la relation entre les normes du travail internationalement reconnues et le système commercial multilatéral. Ce sujet figure dans les conclusions du Président du Comité des négociations commerciales où il est décrit, à l'instar d'autres propositions, comme une suggestion dont l'inclusion dans le programme de travail de l'OMC doit être discutée ${ }^{24}$. A ce jour, l'OMC n'a pas pris de décision sur l'opportunité d'engager des travaux dans ce domaine. Ceci impliquerait la réalisation d'un consensus au sein de ses Membres. Eu égard à la très grande sensibilité politique du sujet et aux réticences des pays en développement, le rapprochement des positions prendra du temps. Dans cette perspective, il importe d'approfondir la réflexion sur la question et de déterminer dans quelle mesure l'OMC est appelée à jouer un rôle dans la promotion du respect universel de certaines normes du travail. A notre sens, deux préalables sont requis pour que l'Organisation aborde cette problématique avec quelque chance d'aboutir à un accord mutuellement acceptable:

- un consensus au sein de l'OIT sur le noyau dur des normes du travail qui doivent être respectées universellement;

- la démonstration qu'il existe effectivement un lien entre les normes du travail et les échanges internationaux.

23. Le mécanisme de règlement des différends de l'OMC autorise une partie plaignante lésée qui aurait obtenu gain de cause à prendre des contre-mesures en vue de rétablir l'équilibre des droits et des obligations. Cependant, les contre-mesures ne sont autorisées qu'en dernier recours, lorsque la partie fautive refuse de se conformer à ses obligations au titre de l'OMC ou qu'elle n'offre pas une compensation acceptable.

24. Cf. GATT FOCUS, Bulletin d'information, Division de l'information et des relations avec les médias du GATT, $\mathrm{n}^{2}$ 107, mai 1994, 5-6. 
Les tâches de l'OIT et de l'OMC doivent être clairement définies. II incombe naturellement à l'OIT d'apporter des réponses au volet normatif de la question; seule l'OIT est en mesure de trouver des solutions fondées sur une volonté universelle associant les partenaires sociaux. L'OMC n'a en effet pas vocation d'élaborer des normes du travail mais de gérer la dimension commerciale d'une éventuelle "clause sociale".

\section{Les travaux de l'Organisation internationale du travail (OIT)}

Avant d'évoquer l'évolution récente, au sein de l'OIT, du débat sur la dimension sociale de la libéralisation du commerce international, il convient de rappeler brièvement les activités déployées par cette organisation en vue d'améliorer la condition des travailleurs. Dans l'esprit des fondateurs, I'OIT a pour mandat d'oeuvrer en faveur de la justice sociale dans le monde, notamment en luttant contre les conditions de travail inhumaines pouvant conduire à des distorsions de concurrence. Ainsi, le préambule de la constitution de l'OIT reconnaît qu'il existe des conditions de travail impliquant pour un grand nombre de personnes l'injustice, la misère et les privations. II précise qu'il est urgent d'améliorer ces conditions, par exemple en ce qui concerne la fixation d'une durée maximum de la journée et de la semaine de travail, la garantie d'un salaire assurant des conditions d'existence convenables, la protection des travailleurs contre les maladies générales ou professionnelles et les accidents résultant du travail, la protection des enfants, des adolescents et des femmes et l'affirmation du principe de la liberté syndicale. Ce même texte rappelle également que la nonadoption par une nation quelconque d'un régime de travail réellement humain fait obstacle aux efforts des autres nations désireuses d'améliorer le sort des travailleurs dans leurs propres pays.

L'OIT a adopté, à ce jour, 176 conventions. Celles-ci font l'objet d'une double lecture, pendant deux années consécutives, au sein d'une commission technique de la Conférence internationale du Travail qui, à l'issue des travaux, adopte formellement les textes proposés. L'une des tâches principales de l'organisation consiste à obtenir que les conventions qu'elle a adoptées soient ratifiées et effectivement appliquées par ses Etats Membres ${ }^{25}$. Elle s'est donc dotée de divers mécanismes de contrôle de même que de procédures pour examiner les plaintes alléguant le non-respect par un Etat d'une convention qu'il a ratifiée. Par ailleurs, une assistance technique est offerte aux gouvernements qui ont besoin de conseils juridiques pour mettre en oeuvre, sur le plan interne, les conventions de l'OIT. Ces dispositifs se sont certes avérés utiles et efficaces dans de nombreux cas, mais leur fonctionnement dépend largement de la volonté politique des Etats Membres. En effet, hormis son «autorité morale", l'OIT ne dispose guère de moyens d'assurer l'application effective de ses

25. Cf. BIT (Bureau international du travail), Defending Values, Promoting Change, Genève, 1994, 29-30. 
conventions. La ratification de ces dernières n'est en aucune façon automatique. On remarquera toutefois que les conventions qui englobent des droits fondamentaux de la personne humaine ${ }^{26}$ sont très largement ratifiées (par plus de 100 Etats Membres). Le tableau présenté ci-après dresse le bilan de la ratification de conventions essentielles de I'OIT dans les pays de l'OCDE et dans quelques pays en développement. Les disparités entre les deux catégories de pays sont importantes et l'on mentionnera que la Chine et la Corée n'ont ratifié aucune de ces conventions alors que les Etats-Unis n'en ont ratifié qu'une (abolition du travail forcé). Les difficultés afférentes à la ratification des conventions de l'OIT tiennent parfois au fait qu'elles sont formulées en termes trop détaillés et que certains Etats qui en respectent les principes généraux ne sont pas en mesure de se conformer à des dispositions relativement mineures. A l'inverse, le fait qu'un Etat ratifie une convention ne signifie pas qu'il appliquera scrupuleusement les dispositions qu'elle contient.

En juin 1994, soit peu après l'achèvement du cycle d'Uruguay, le Conseil d'administration du Bureau international du travail (BIT) a décidé de mettre sur pied un groupe de travail pour traiter de tous les aspects pertinents de la dimension sociale de la libéralisation des échanges. Ce groupe de travail a siégé en novembre 1994 et en mars-avril 1995. II a débattu de l'opportunité d'introduire dans le système commercial multilatéral des garanties minimales permettant à l'ensemble des acteurs du champ économique de tirer profit de la croissance découlant de la libéralisation du commerce international. II a aussi examiné les initiatives plus spécifiques que I'OIT devrait prendre pour faire en sorte que le progrès social et une plus grande ouverture aux échanges s'épaulent mutuellement. A l'origine, l'exercice avait pour objectif d'aider à la réflexion et de déterminer dans quelle mesure il y avait un besoin d'action spécifique de la part de la communauté internationale. Eu égard à la très grande sensibilité politique de la question, le débat, greffé sur une opposition Nord-Sud, s'est très rapidement transformé en un affrontement mettant aux prises partisans et adversaires de la "clause sociale". En dépit des nombreuses divergences, il a tout de même été possible de dégager les éléments de consensus suivants:

- le développement économique et le progrès social sont largement interdépendants; lorsque le développement économique est peu avancé, le niveau de protection sociale est également moins élevé; c'est la raison pour laquelle les pays en développement ont des normes du travail moins sévères; leurs conditions sociales devraient s'améliorer avec la croissance économique et la libéralisation des échanges bien que cette relation ne soit pas automatique;

26. On considérera qu'il s'agit des conventions relatives à l'interdiction du travail forcé (Conventions 29 et 105), à la liberté syndicale (Convention 87), au droit d'organisation et de négociation collective (Convention 98) et à la non-discrimination dans l'emploi (Convention 111). 
Ratification de conventions essentielles de l'OIT (Etat au 31 décembre 1993)

\begin{tabular}{rrrrrr}
\hline $\begin{array}{r}\text { Liberté } \\
\text { syndicale }\end{array}$ & $\begin{array}{r}\text { Droit de } \\
\text { négociation } \\
\text { collective }\end{array}$ & $\begin{array}{r}\text { Travail } \\
\text { forcé }\end{array}$ & $\begin{array}{r}\text { Abolition } \\
\text { du travail } \\
\text { forcé }\end{array}$ & $\begin{array}{r}\text { Egalité de } \\
\text { rémuné- } \\
\text { ration entre } \\
\text { femme et } \\
\text { homme }\end{array}$ & $\begin{array}{r}\text { Suppression } \\
\text { de discrimi- } \\
\text { nations } \\
\text { dans }\end{array}$ \\
(Conv. 87) & (Conv.98) & (Conv.29) & l'emploi \\
(Conv.105) & (Conv.100) & (Conv.111) \\
\hline
\end{tabular}

\section{Hors OCDE}

\begin{tabular}{|c|c|c|c|c|c|c|}
\hline Argentine & $r$ & $r$ & $r$ & $r$ & $r$ & $r$ \\
\hline Brésil & & $r$ & $r$ & $r$ & $r$ & $r$ \\
\hline Chili & & & $r$ & & $r$ & $r$ \\
\hline $\begin{array}{l}\text { Chine } \\
\text { Hong Kong }\end{array}$ & & & & & $r$ & \\
\hline Inde & & & $r$ & & $r$ & $r$ \\
\hline Indonésie & & $r$ & $r$ & & $r$ & \\
\hline Corée & & & & & & \\
\hline Malaisie & & $r$ & $r$ & $r$ & & \\
\hline Maroc & & $r$ & $r$ & $r$ & $r$ & $r$ \\
\hline Philippines & $r$ & $r$ & & $r$ & $r$ & $r$ \\
\hline Singapour & & $r$ & $r$ & $r$ & & \\
\hline Thaïlande & & & $r$ & $r$ & & \\
\hline
\end{tabular}

\section{OCDE}

\begin{tabular}{|c|c|c|c|c|c|c|}
\hline Australie & $r$ & $r$ & $r$ & $r$ & $r$ & $r$ \\
\hline Allemagne & $r$ & $r$ & $r$ & $r$ & $r$ & $\mathrm{r}$ \\
\hline Autriche & $r$ & $r$ & $r$ & $r$ & $r$ & $r$ \\
\hline Belgique & $r$ & $r$ & $r$ & $r$ & $r$ & $r$ \\
\hline Canada & $r$ & & & $r$ & $r$ & $r$ \\
\hline Danemark & $r$ & $r$ & $r$ & $\mathbf{r}$ & $r$ & $\mathrm{r}$ \\
\hline Espagne & $r$ & $r$ & $r$ & $r$ & $r$ & $r$ \\
\hline Etats-Unis & & & & $r$ & & \\
\hline Finlande & $r$ & $r$ & $r$ & $r$ & $r$ & $r$ \\
\hline France & $r$ & $r$ & $r$ & $r$ & $r$ & $\mathrm{r}$ \\
\hline Grèce & $r$ & $r$ & $r$ & $r$ & $r$ & $\mathrm{r}$ \\
\hline Islande & $r$ & $r$ & $r$ & $r$ & $r$ & \\
\hline Italie & $r$ & $r$ & $\mathbf{r}$ & $r$ & $r$ & $r$ \\
\hline Japon & $r$ & $r$ & $r$ & & $r$ & \\
\hline Luxembourg & $r$ & $r$ & $r$ & $r$ & $\mathrm{r}$ & \\
\hline Mexique & $r$ & & $\mathbf{r}$ & $r$ & $r$ & $r$ \\
\hline Nvlle Zélande & & & $r$ & $r$ & $r$ & $r$ \\
\hline Norvège & $r$ & $r$ & $r$ & $r$ & $\mathrm{r}$ & $\mathrm{r}$ \\
\hline Pays Bas & $r$ & $r$ & $r$ & $r$ & $\mathrm{r}$ & $\mathrm{r}$ \\
\hline Portugal & $r$ & $r$ & $\mathbf{r}$ & $r$ & $r$ & $r$ \\
\hline Royaume Uni & $r$ & $r$ & $\mathbf{r}$ & $r$ & $r$ & \\
\hline Suéde & $r$ & $r$ & $r$ & $r$ & $r$ & $\mathrm{r}$ \\
\hline Suisse & $r$ & & $\mathbf{r}$ & $r$ & $r$ & $\mathrm{r}$ \\
\hline Turquie & $r$ & $r$ & & $r$ & $r$ & $\mathrm{r}$ \\
\hline
\end{tabular}

Source: OIT, Listes des ratifications par convention et par pays, Rapport III (Partie 5), Conférence du Travail, 81e session, 1994. 
- les pays en développement ont un droit légitime de mettre à profit l'avantage comparatif que représentent des coûts de main-d'oeuvre moins élevés; les droits de l'homme ou les normes sociales ne doivent pas servir de prétextes au protectionnisme; cependant, aucun pays ne peut délibérément recourir à l'oppression, au travail forcé ou à la discrimination dans l'emploi pour compresser artificiellement les coûts de production;

- le rôle de l'OIT ne doit pas être en priorité de prendre des sanctions dans le but de promouvoir des normes du travail plus élevées; elle s'appuiera sur les mesures d'assistance et d'encouragement; il est en particulier reconnu que le travail des enfants ne pourra pas être aboli dans de brefs délais;

- les Etats Membres sont unanimes à reconnaître qu'il faut affermir la capacité des pays à améliorer leurs normes sociales et à appliquer effectivement les instruments de l'OIT; les mécanismes de contrôle de la mise en oeuvre des normes et la coopération internationale doivent être renforcés;

- le groupe de travail suspendra toute discussion sur la relation entre les échanges et les normes sociales de même que sur les moyens d'assurer le respect d'une "clause sociale" par le biais d'un mécanisme de sanctions commerciales.

Ces points de convergence reflètent la teneur générale du débat mais ne constituent en aucune façon des résolutions formellement adoptées. A ce stade, I'OIT n'est donc pas parvenue à identifier le noyau dur des droits fondamentaux des travailleurs qui doivent être respectés universellement. Le débat international sur la question laisse toutefois entrevoir les contours d'un socle social minimal. La question a d'ailleurs été abordée à l'occasion du Sommet mondial pour le développement social qui s'est tenu en mars 1995 à Copenhague. On y a retenu les principes suivants dont certains relèvent des droits fondamentaux de la personne humaine: le droit à la liberté syndicale, le droit de négociation collective, l'interdiction du travail forcé, l'interdiction de la discrimination dans l'entreprise et la profession, le droit à une rémunération égale entre femmes et hommes et l'interdiction du travail des enfants. II a été constaté que I'OIT, par son mandat, sa structure tripartite et son expertise, devait jouer un rôle essentiel dans la mise en oeuvre des décisions prises à Copenhague.

Les impulsions du Sommet mondial pour le développement social sont de nature à insuffler un dynamisme nouveau aux travaux de l'OIT. II y aurait ainsi lieu d'examiner dans quelle mesure les conventions de l'OIT recouvrant les principes énoncés à Copenhague sont formulées d'une manière qui favorise leur application universelle. Le cas échéant, il s'agirait d'en concevoir de nouvelles versions simplifiées, sans pour autant que la protection accordée ne soit ramenée à un niveau inférieur. L'Organisation pourrait également étudier les possibilités de renforcer les moyens dont elle dispose pour assurer l'application effective des normes du travail fondamentales auxquelles ses Etats Membres ont souscrit. II conviendrait ici de tabler sur des mesures positives telles que la promotion de la ratification des normes, la coopération technique et les activités de conseil, ainsi que de renforcer les mécanismes de traitement des plaintes déposées auprès des organes de contrôle de l'OIT. En outre, il y aurait lieu de 
déterminer si ces mécanismes sont suffisamment efficaces ou s'il est nécessaire de prévoir d'autres dispositifs. Ce programme paraît ambitieux mais il s'inscrit dans l'esprit des textes de base de l'OIT dont l'objectif est de promouvoir la justice sociale. II est donc souhaitable que ces travaux débouchent sur des résultats tangibles propres à améliorer la condition des travailleurs dans le plus grand nombre de pays. Cependant, il n'appartient pas à l'OIT de juger de l'opportunité de recourir à des mesures commerciales pour assurer l'application effective des normes du travail fondamentales.

Afin d'éviter des malentendus qui pourraient aviver les tensions entre les partenaires sociaux et les pays, il importe toutefois d'approfondir la réflexion sur la véritable nature de la relation entre le commerce et les normes du travail fondamentales. Dans le contexte d'une économie de plus en plus globalisée, l'ouverture aux échanges nuit-elle gravement à la protection sociale ou, au contraire, entraîne-t-elle une amélioration générale de la condition des travailleurs? Nous l'avons signalé en introduction, les répercussions concrètes de la libéralisation des échanges sur le plan social ne sont pas aisées à cerner. L'étude exécutée actuellement par l'OCDE nous permettra certainement de mieux comprendre le phénomène et constituera un élément de réponse important. Nous nous proposons ci-après de présenter certaines constatations issues de recherches consacrées à ce thème et d'en tirer quelques conclusions préliminaires.

\section{Globalisation, libéralisation des échanges et normes du travail}

\subsection{L'impact des échanges internationaux sur la situation de la main- d'oeuvre des pays développés}

La libéralisation du commerce international n'est pas un jeu à somme nulle: la création d'échanges et de revenu qui en résulte bénéficie globalement à la collectivité. Cependant, l'ouverture à l'échange ne transmet pas instantanément ses avantages à l'ensemble des agents économiques; pour en tirer profit, les catégories de travailleurs les moins compétitives au plan international doivent parfois consentir d'importants efforts d'adaptation. Elles doivent également renoncer à la rente que les mesures protectionnistes leur procuraient. La répartition des gains de l'échange international entre les diverses strates de qualification professionnelle peut être analysée à l'aide d'un modèle théorique: le théorème d'égalisation du prix des facteurs. Ce modèle prédit que l'élimination des entraves au commerce réduit le revenu relatif du facteur de production utilisé intensivement dans les produits importés et qu'elle augmente le revenu relatif du facteur de production utilisé intensivement dans les exportations. Ainsi, dans les pays industrialisés, la libéralisation des échanges avec les pays en développement augmenterait la demande et, partant, le prix relatif du bien manufacturé dont la production est intensive en main-d'oeuvre qualifiée. Le salaire relatif de la main-d'oeuvre qualifiée s'accroîtrait, alors que celui de la main-d'oeuvre peu qualifiée diminuerait: en effet, la main-d'oeuvre peu qualifiée des pays indus- 
trialisés étant en concurrence avec celle des pays en développement dont le coût est inférieur, le travailleur peu qualifié des pays industrialisés doit diminuer le prix auquel il vend ses services afin de rester compétitif. Pour forcer le trait, le théorème prévoit que la rémunération du travail - à niveau de qualification égal - tend à s'égaliser dans les régions où l'intégration économique est la plus poussée. Les conditions de réalisation de cet aboutissement extrême ne sont toutefois pas réunies au niveau mondial. D'abord, les hypothèses de base ${ }^{27}$ très strictes du théorème d'éaglisation du prix des facteurs ne sont pas vérifiées dans la réalité économique. Ensuite, quand bien même le processus de globalisation de la production est une tendance lourde, nous ne vivons pas sous le régime d'une économie unifiée. II sera vraisemblablement impossible de supprimer tous les obstacles techniques, culturels ou naturels qui segmentent les marchés. Les études empiriques démontrent d'ailleurs que, sur l'ensemble de la planète, les divergences s'accentuent dans le temps: le rapport du revenu par tête des pays les plus riches à celui des pays les plus pauvres a passé de 11 en 1870, à 38 en 1960 et à 52 en $1985^{28}$. En revanche, on observe une convergence partielle des revenus en Europe et aux Etats-Unis où l'intégration économique est plus profonde et les conditions de départ similaires alors que les économies dynamiques d'Asie comblent progressivement leur retard. II est impossible de prédire aujourd'hui si la globalisation supprimera à terme les inégalités entre les pays industrialisés et les pays les moins avancés sous l'effet d'une convergence des revenus par le haut ou par le bas.

Dans les pays de l'OCDE, on a toutefois constaté, depuis la fin des années 1970, une réaffectation de la main-d'oeuvre, la demande de travail se déplaçant des emplois non qualifiés vers des emplois plus qualifiés. Parallèlement, les écarts de revenu entre travailleurs qualifiés et travailleurs non qualifiés se sont creusés en Australie, au Canada, aux Etats-Unis et dans le Royaume-Uni. Cette accentuation des inégalités de gains a été accompagnée d'une réduction des salaires réels de la main-d'oeuvre de faible qualification dans les trois premiers pays. En revanche, dans les pays d'Europe continentale, les écarts de revenus sont demeurés constants ou n'ont que légèrement augmenté alors que la plus grande rigidité des salaires réels y entraînait une dégradation des taux d'emploi ${ }^{29}$. D'aucuns imputent ce phénomène, comme le suggère le théorème d'égalisation du prix des facteurs, à l'essor du commerce avec les pays en développement où le travail est faiblement rémunéré. Adrian Wood ${ }^{30}$ (Sussex

27. Le théorème postule notamment que la concurrence est parfaite, les biens, de même que les préférences des consommateurs sont homogènes, les fonctions de production sont identiques et linéaires (pas d'économies d'échelle) dans les divers pays et les facteurs de production n'entrent pas dans l'échange international.

28. Cf. The World Bank, op. cit., 53.

29. Cf. OCDE, L'étude de l'OCDE sur l'emploi. Faits. Analyses. Stratégies, Synthèse, Paris, 1994, 22-24.

30 Cf. Wood A., North-South Trade, Employment and Inequality, Oxford, Clarendon Press, 1994. 
University) défend que l'accroissement des échanges avec les pays en développement aurait réduit, jusqu'en 1990, la demande de travail non qualifié de $20 \%$ dans les pays industrialisés. Ce déclin se serait produit, pour les trois quarts, dans les années 1980 et serait à l'origine d'une plus grande disparité des salaires aux Etats-Unis et en Grande-Bretagne et d'une aggravation du chômage en Europe continentale. L'auteur soutient que le faible taux de pénétration des importations en provenance des pays en développement (moins de quatre pour cent du produit national brut des pays industrialisés) n'est pas incompatible avec ses résultats. Ces importations se concentrent en effet dans des secteurs à fort coefficient de main-d'oeuvre où les pertes d'emploi dues à la concurrence internationale et à la rationalisation de la production sont relativement nombreuses. Selon Adrian Wood, l'effet direct du commerce ne compte que pour un quart de la rétraction de $20 \%$ de la demande de travailleurs peu qualifiés, le solde étant notamment imputé aux innovations techniques découlant de la concurrence internationale et qui ont pour effet d'économiser la maind'oeuvre. A l'extrême opposé, on peut citer l'étude de Robert Lawrence et Matthew Slaughter ${ }^{31}$ qui ont entre autres constaté qu'aux Etats-Unis le prix des biens dont la production est intensive en main-d'oeuvre faiblement qualifiée s'était accru par rapport aux biens dont le contenu en travail qualifié est important. Ce résultat est incompatible avec le théorème de Stolper-Samuelson. Pour ces économistes, la diminution de la demande de travailleurs peu qualifiés s'explique essentiellement par les changements technologiques intervenus au cours des années 1980 .

Ces deux thèses sont contestées. Certains chercheurs ${ }^{32}$ considèrent en effet qu'Adrian Wood a exagéré l'impact du commerce avec les pays en développement sur la demande de travail non qualifié dans les pays industrialisés. Ils relèvent cependant que le progrès technologique n'est pas le seul facteur en cause et qu'une partie des changements observés sur le marché de l'emploi revient effectivement aux échanges avec les pays en développement. Ceci étant, il ne faut pas oublier que les pays de l'OCDE font l'essentiel de leur commerce entre eux ${ }^{33}$ et que la concurrence à laquelle ils doivent faire face vient avant tout de la zone OCDE et non pas des pays à bas salaires. La majorité de ces échanges s'effectuent à l'intérieur d'un même secteur ou entre

31. Lawrence R. et Slaughter M., International Trade and American Wages in the 1980s: Giant Sucking Sound or Small Hiccup, Brookings Papers on Economic Activity, 1993.

32. Cf. Sachs J. et Shatz H., Trade and Jobs in US Manufacturing, Brookings Papers on Economic Activity, 1994.

33. En 1992, les importations en provenance des pays hors OCDE ne représentaient que $3,8 \%$ du PIB des pays de l'OCDE (Cf. OCDE, L'étude de l'OCDE sur l'emploi. Données et explications. Partie I. Évolution des marchés du travail et facteurs de changement, Paris, 1994, 85-86). Selon la CNUCED (Manuel de statistiques du commerce international et du développement), la part des exportations à l'intérieur de la zone OCDE a augmenté au cours des trois dernières décennies, passant de $69,3 \%$ en $1958-60$ à $76,5 \%$ en $1990-91$. 
entreprises (produits semi-finis), ou portent sur des produits similaires fabriqués par une main-d'oeuvre de qualification homogène. Emanation de la globalisation des économies, ce phénomène requiert une grande capacité d'adaptation face aux progrès techniques intervenus dans tous les secteurs et en particulier face à l'avènement des nouvelles technologies de l'information ${ }^{34}$. Le marché du travail s'en trouve profondément affecté: l'OCDE estime qu'environ un emploi sur dix est détruit chaque année et qu'il s'en crée un nombre équivalent. Dans les pays industrialisés, les disparitions d'emplois concernent en premier lieu les secteurs à faible niveau technologique et à fort coefficient de main-d'oeuvre (textile, habillement, chaussure, électronique grand public par exemple) alors que les gisements de travail recouvrent principalement le domaine des services et quelques industries manufacturières de pointe telles que l'informatique, la bureautique, les télécommunications, les semi-conducteurs et l'aérospatiale. Pour bénéficier des ajustements positifs du redéploiement de la production, les travailleurs défavorisés par le progrès technique et la libéralisation des échanges se doivent de faire preuve d'une grande aptitude à la reconversion professionnelle. Pendant la phase d'adaptation, cette catégorie sera particulièrement vulnérable au chômage.

Il est donc malaisé, dans un contexte de mondialisation de la production, de similitude dans l'échange international et de progrès technique rapide d'isoler la part qui revient au commerce avec les pays en développement dans l'explication des disparités de salaires et du chômage observés dans les pays industrialisés. L'OCDE est parvenue à la conclusion que cette part était faible au niveau macro-économique ${ }^{35}$ et que d'autres facteurs, en particulier les améliorations techniques indépendantes des ajustements dus à la concurrence des pays à bas salaires, jouaient un rôle plus déterminant ${ }^{36}$. L'organisation a en outre mis en évidence certains phénomènes compensatoires immédiats, notamment le fait que la croissance des exportations en provenance du Sud, en particulier des pays à bas salaires d'Asie, est allée de pair avec un essor comparable des exportations de la zone OCDE à destination de ces mêmes pays.

Si l'incidence de l'intensification des échanges avec les pays en développement sur le marché de l'emploi des pays de l'OCDE est faible, qu'en est-il de celle des normes du travail, qui ne constituent qu'une composante des coûts de production? Le fait que le coût horaire d'un travailleur est de 25 dollars en Allemagne, de 16 dollars aux Etats-Unis, de 5 dollars en Corée du Sud, de 2,4 dollars au Mexique et de 0,5 dollar ou moins en Chine, en Inde et en Indoné-

34. Cf. OCDE, Technology and the Economy: The Key Relationships, Paris, 1992.

35. La Banque Mondiale parvient également à la conclusion que l'incidence du commerce avec les pays en développement est modeste en regard des autres facteurs qui affectent le marché de l'emploi dans les pays industrialisés (Cf. The World Bank, op. cit., 56).

36. OCDE, L'étude de l'OCDE sur l'emploi. Données et explications. Partie I. Évolution des marchés du travail et facteurs de changement, Paris, 1994, 83-118. 
$\mathrm{sie}^{37}$ ne s'explique que marginalement par le respect ou le non-respect de normes fondamentales du travail. La rémunération de la main-d'oeuvre est avant tout fonction des conditions du marché du travail et du niveau de développement économique. II ne fait pas de doute que l'offre pléthorique de maind'oeuvre des pays en développement et la faible productivité globale de leurs économies y dépriment naturellement les salaires réels. A supposer que ces pays appliquent effectivement les mêmes normes fondamentales du travail que les pays industrialisés, on ne peut s'attendre à ce que s'y produise un accroissement notable des salaires qui serait incompatible avec leurs profils économiques de base. Ils conserveraient largement l'avantage comparatif dont ils bénéficient dans les secteurs intensifs en travail de faible qualification. Au niveau global, les mécanismes du marché semblent façonner de manière déterminante la performance du commerce extérieur. On ne saurait donc imputer la détérioration de la situation de la main-d'oeuvre peu qualifiée des pays industrialisés à l'application de normes du travail médiocres dans les pays en développement.

\subsection{Développement économique, intégration dans l'échange internatio- nal et normes du travail}

La Banque mondiale a établi, sur un échantillon de 22 pays à revenu faible ou intermédiaire, qu'une croissance durable du produit national brut était positivement corrélée avec un accroissement du salaire réel dans les secteurs agricole et manufacturier ${ }^{38}$. Le développement économique permet aux travailleurs de trouver de l'embauche dans des secteurs à plus forte productivité et, partant, plus rémunérateurs. Ces résultats indiquent que la condition des travailleurs et le degré de développement sont étroitement interdépendants et que l'on ne saurait attendre que les pays les plus pauvres se dotent de régimes sociaux aussi ambitieux et onéreux que ceux des pays les plus avancés. Par exemple, il n'est pas étonnant que l'assurance sociale dans le domaine de la santé couvre $90 \%$ de la population en Corée du Sud alors qu'elle n'en couvre que $13 \%$ en Indonésie, $10 \%$ au Kenya et $5 \%$ en Inde ${ }^{39}$. II serait vain d'exiger d'un Etat qu'il applique des normes du travail qui lui sont inaccessibles car incompatibles avec son niveau de développement économique. En guise d'illustration, on évoquera ci-dessous la fixation d'un âge minimal pour exercer un emploi.

L'OIT estime qu'environ $18 \%$ des enfants âgés de 10 à 14 ans travaillent régulièrement. Une petite fraction de ces derniers sont engagés dans le secteur structuré tourné vers l'exportation. L'écrasante majorité travaille dans des secteurs informels: exploitations agricoles familiales, petit commerce et services. ${ }^{40}$

37 Cf.. "A Survey of the global Economy", War of the World, October 1994, 16.

38 Cf. The World Bank, op. cit., 16-22.

39 Cf. Bureau international du travail, Le travail dans le monde, Genève, 1994, 77.

40 Cf. Bonnet M., "Child Labour in Africa", International Labour Review, vol. 132, 1993, 371-389; Black M., In the Twilight Zone. Child Workers in the Hotel, Tourism and Catering Industry, International Labour Office, Geneva, 1995. 
Dans les zones rurales des pays en développement, les parents ne peuvent se passer du concours de leurs enfants que pendant la saison morte. Lors des récoltes ou des semailles, ils renonceront donc à les envoyer à l'école pour leur faire exécuter des travaux souvent longs et harassants. Au siècle passé, cette attitude était également répandue dans les campagnes suisses quand bien même le principe de l'enseignement primaire obligatoire s'était largement affirmé dans les législations cantonales et était ancré dans la Constitution fédérale de $1874 .{ }^{41}$ Des structures de production traditionnelles et la pauvreté étaient à l'origine de contraintes qui faisaient obstacle à la généralisation de la scolarisation et à l'élimination du travail des enfants. La situation s'est nettement améliorée au tournant du XXe siècle, avec l'essor économique qu'a connu la Confédération. II en va de même pour les pays en voie de développement aujourd'hui: dans les économies dynamiques du Sud-Est asiatique, l'emploi des enfants est nettement plus rare qu'en Ethiopie, au Burkina Faso, au Burundi, en Inde ou au Bangladesh. En Egypte, un accroissement de 10\% du salaire des mères induit une contraction de $15 \%$ du travail des enfants âgés de douze à quatorze ans et un recul de $27 \%$ du travail des enfants âgés de six à onze ans. ${ }^{42}$ Des mesures prises pour abaisser le coût de la scolarisation contribuent également à faire diminuer la proportion de main-d'oeuvre enfantine. On le voit, l'incidence de la pauvreté est déterminante dans l'explication de l'emploi des enfants. En décréter l'interdiction, en l'absence des conditions économiques nécessaires pour qu'un tel objectif puisse se réaliser sans effets pervers, peut entraîner une détérioration de la situation des enfants. L'OIT a constaté, dans certains pays en développement, que la condition des enfants pouvait se dégrader si, pour se plier à des pressions externes, le gouvernement les privait de la possibilité d'exercer une activité lucrative. II arrive en effet que l'apport des enfants constitue la seule source de revenu légal d'une famille; privé de ces gains, un enfant est parfois réduit à la mendicité, à la prostitution ou encore à la famine $e^{43}$. Ceci ne justifie évidemment pas les servitudes les plus extrêmes (travail nuisant gravement à la santé par exemple). L'effort de la communauté internationale et des gouvernements doit précisément contribuer à supprimer ces formes d'exploitation par l'établissement d'une réglementation appropriée et de programmes d'incitation ou d'assistance ${ }^{44}$. L'OIT a d'ailleurs fait une priorité de cette question. En revanche, le recours aux sanctions commerciales pourrait produire des effets indésirables dans la mesure où la participation à l'échange international

41. R. Métrailler qui a étudié le cas du Valais considère que l'école obligatoire "s'annonce comme l'ennemi numéro un de l'entreprise familiale" au XIXe siècle. (Cf. Métrailler R., L'école primaire en Valais durant la deuxième partie du 19ème siècle et son processus de popularisation jusqu'à la veille de la première guerre mondiale, Mémoire de licence, Fribourg, 1978).

42. Cf. The World Bank, op. cit., 72.

43. Cf. The Economist, op. cit., 41.

44. Bequele A. and Myers W.E., First Things, First in Child Labour. Eliminating Work Detrimental to Children, International Labour Office, Geneva, 1995. 
est l'un des moteurs du développement économique qui est lui-même à la base de l'amélioration des normes du travail.

On a pu entendre dans le débat public que certains pays en développement appliquaient des normes du travail artificiellement basses pour accentuer leurs avantage comparatifs et gagner des parts du marché international. Ce phénomène devait être particulièrement marqué dans les économies émergentes dont les exportations se sont rapidement accrues depuis le début des années 1980. Nous allons examiner ci-après dans quelle mesure l'évolution récente des profils sociaux et économiques de ces régions permet d'accréditer cette assertion. En guise de préambule, nous voudrions rappeler que l'amélioration de la condition des travailleurs ne découle pas nécessairement de la ratification des conventions de I'OIT. Les Etats optent quelquefois pour une approche indirecte qui consiste à favoriser le développement économique et les mécanismes du marché ${ }^{45}$. Ils tablent ainsi sur le jeu de l'offre et de la demande pour améliorer la condition des travailleurs et renoncent à instituer des normes ambitieuses. Cette démarche a prédominé dans certains pays d'Extrême-Orient tels que Singapour, Hong-Kong, la Corée du Sud et Taiwan qui ont remarquablement profité de la participation à l'échange international. L'un des effets que l'on attend du respect des droits d'organisation et de négociation collective est une distribution plus équitable des gains de la croissance économique. L'approche indirecte s'est-elle révélée efficace? De 1980 à 1990, les salaires réels ont quasiment doublé dans les quatre économies sous revue et le chômage y est tombé à des taux très bas (de l'ordre de $2 \%$ ). On observe aussi que la croissance du produit intérieur brut par habitant et la progression des gains du travail y ont évolué à un rythme pratiquement similaire durant la même période. De plus, à la différence d'autres pays en développement comme le Brésil ou la Jamaïque, ces pays se singularisent par des disparités de rémunération relativement faibles entre le haut et le bas de l'échelle des revenus ${ }^{46}$. Ces résultats attestent la réalisation d'un effet de distribution. Dans les quatre pays en question, la consolidation du développement économique a retenti positivement sur les relations professionnelles et les droits des travailleurs. L'exemple de la Corée du Sud est particulièrement révélateur à cet égard. La pratique de la négociation collective tend à s'y institutionnaliser, généralement sous une forme décentralisée au niveau des entreprises. Des systèmes de protection sociale s'y sont créés. Le droit d'association s'y est affermi et le nombre d'organisations de travailleurs, dont la plupart sont des syndicats internes aux entreprises, y a passé de 2742 en 1988 à 7698 en $1992^{47}$. Il convient encore de signaler qu'une corrélation positive entre l'intégration dans l'échange international et le revenu du travail ne s'observe pas uniquement dans les économies dynamiques d'Asie.

45. Cf. OCDE, Échanges et normes du travail. Examen des principales questions, OCDE, Paris, 1995, 17-18.

46. Cf. OCDE, Échanges et normes du travail. Examen des principales questions, OCDE, Paris, 1995, 19-21.

47. Cf. The World Bank, op. cit., 84. 
Au cours des deux dernières décennies, les salaires réels du secteur manufacturier ont augmenté à un taux annuel moyen de $3 \%$ dans les pays en développement où le rapport de la croissance des exportations au PNB était supérieur à la médiane $^{48}$ alors qu'ils stagnaient dans les pays où les exportations s'accroissaient le moins ${ }^{49}$. Contrairement à l'opinion que nous avons évoquée plus haut, cette relation donne à penser que l'essor du commerce et l'amélioration de la condition des travailleurs peuvent être positivement corrélés.

Néanmoins, cette spirale vertueuse ne prend pas instantanément forme et il existe des situations où, durant la phase de décollage surtout, des pays en développement restreignent les droits des travailleurs dans l'intention de réduire les coûts de production et d'attirer les investissements étrangers. II arrive que pareilles restrictions constituent l'un des éléments du traitement préférentiel accordé aux entreprises implantées dans les zones franches industrielles travaillant pour l'exportation. Ces mesures viennent s'ajouter à d'autres avantages $^{50}$ favorisant l'investissement car une réglementation du travail souple est, dans la plupart des cas, insuffisante pour attirer les investissements étrangers. D'abord, il n'est pas acquis qu'elle réduise le coût de la main-d'oeuvre, celui-ci étant aussi influencé par la dotation du pays en travailleurs. Ensuite, le coût de la main-d'oeuvre n'est pas le seul facteur qui détermine la localisation des investissements directs; il n'est décisif que pour les biens manufacturés pour lesquels la production est intensive en travail (secteurs ${ }^{51}$ de la chaussure et du textile par exemple). Or, depuis les années septante, le rapport du coût de la main-d'oeuvre aux coûts totaux de production a diminué de $25 \%$ à $5-10 \%$ dans de nombreuses industries des économies développées. ${ }^{52}$ Le capital, la technologie, la proximité des marchés et l'infrastructure sont devenus des déterminants de la compétitivité de plus en plus importants au détriment du coût de la main-d'oeuvre. On constate d'ailleurs que les investissements directs des pays industrialisés vers les pays à bas salaires, qui ont augmenté dans les années 1980 , se sont stabilisés au début des années 1990. Depuis 1990, les sorties nettes de capitaux n'ont réduit les capitaux disponibles des pays industrialisés que de $0,5 \% .{ }^{53}$ Sur cette toile de fond, une étude de la CNUCED conclut que

48. On appelle médiane une valeur telle que le nombre des observations qui lui sont inférieures soit égal au nombre des observations qui lui sont supérieures dans un ensemble. Dans le cas d'espèce, $50 \%$ des pays en développement ont un rapport de la croissance des exportations au PNB supérieur à la médiane et $50 \%$ un rapport inférieur.

49. Cf. The World Bank, op. cit., 54-55.

50. En matière de régimes douanier, fiscal et d'exportation de devises par exemple

51. Dans les industries des textiles et de la chaussure la part du coût de la maind'oeuvre dans les coûts totaux de production équivaut encore à $30 \%$.

52. Cf. Oman C., Globalisation and Regionalisation: The Challenge for Developing Countries, OECD, Paris, 1994.

53. Cf. Krugman P., "Does Third World Growth hurt First World Prosperity?", Harvard Business Review, July-August, 1994. 
l'existence d'une corrélation entre la localisation des investissements directs à l'étranger et la rigueur de la législation du travail est très improbable. ${ }^{54}$

II n'en demeure pas moins que les zones franches ont joué un rôle important dans l'essor des exportations des pays asiatiques. On en dénombre aujourd'hui plus de 500 dans le monde dont une grande partie est située en Asie et plus particulièrement en Chine. Dans des pays où les politiques de libéralisation des échanges sont récentes, on a constaté que certains droits des travailleurs reconnus au niveau national n'étaient pas appliqués ou restreints dans les zones franches, notamment en matière de liberté syndicale (République dominicaine, Philippines, Bangladesh, Pakistan, Turquie, Jamaïque, Sri Lanka, Malaisie, par exemple). Il existe également des contre-exemples comme celui du Mexique où, dans le cadre du programme Maquiladoras, les normes du travail tendent à s'aligner sur celles des Etats-Unis ${ }^{55}$. En fait, les politiques appliquées dans les zones franches, de même que le traitement des travailleurs qui y trouvent de l'embauche, varient sensiblement d'un pays à l'autre. Généralement, la situation s'améliore au gré du développement économique et l'on note qu'une réglementation sociale souple ne va pas nécessairement de pair avec de mauvaises conditions de travail. Selon l'OIT, les zones franches se caractérisent en effet par une augmentation de l'emploi, des rémunérations et des conditions de travail "égales ou supérieures à celles qu'offre leur environnement immédiat ${ }^{56}$ ').

II convient également de préciser que les entreprises multinationales sont les principaux investisseurs dans les zones franches. ${ }^{57}$ Ces dernières, dont les activités sont très exposées à l'observation du public et des médias, ne peuvent pas se livrer, dans leurs entreprises des zones franches, à une exploitation de la main-d'oeuvre qui susciterait l'indignation. II existe d'ailleurs des codes de conduite volontaires invitant les entreprises multinationales à respecter certaines normes du travail comme le droit de représentation des salariés. On mentionnera ici les Principes directeurs de la Déclaration sur l'investissement international et les entreprises multinationales de l'OCDE $^{58}$, la Déclaration tripartite de l'OIT et le Code international de conduite relatif aux sociétés transnationales de l'Organisation des Nations Unies. Si ces instruments ne sont pas juridiquement contraignants, ils sont tout de même assortis de procédures de suivi dans

54. UNCTAD, World Investment Report 1994, New York and Geneva.

55. Cf. United States General Accounting Office, U.S.-Mexico Trade: The Work Environment at Eight U.S.-Owned Maquiladora Auto Parts Plants, November 1993.

56. Cf. Bureau international du travail, L'emploi dans le monde. Un rapport du BIT, Genève, 1995, 83.

57. Le BIT estime que les firmes étrangères représentent $63 \%$ de l'ensemble des firmes implantées dans les zones franches aux Philippines, $77 \%$ en Corée et plus de $80 \%$ en République dominicaine, au Sri Lanka, en Jamaïque et au Mexique (Cf. ILO, Multinational and Employment, Geneva, 1993).

58. Cf. OCDE, Principes directeurs à l'intention des entreprises multinationales, Paris, 1994. 
le cadre desquelles les problèmes afférents à leur application peuvent être soulevés. Ces examens favorisent également le dialogue entre les partenaires sociaux. L'application de la Déclaration de l'OIT fait l'objet d'enquêtes triennales dont la plus récente date de 1992. Cette dernière "constate que la Déclaration de principe est assez largement respectée. On a observé que les entreprises multinationales respectaient généralement la législation nationale et appliquaient souvent, en matière de salaires, prestations, conditions de travail et sécurité et hygiène, des normes supérieures aux exigences légales et à la pratique des entreprises locales ${ }^{59}$ ». Certaines offrent même des conditions plus avantageuses non seulement à leur propre personnel mais aussi à celui de leurs fournisseurs dans le but d'améliorer leur image et d'attirer les meilleurs travailleurs locaux. En outre, de nombreuses filiales à l'étranger appliquent les mêmes normes du travail que celles en vigueur dans le pays de l'entreprisemère. ${ }^{60}$ L'International Metalworkers' Federation (IMF) est aussi parvenue à la conclusion que la forte présence des multinationales dans les zones franches contribue à y améliorer les conditions de travail en regard de celles qui prévalent en moyenne nationale. ${ }^{61}$

Ce bilan positif s'entend évidemment de tendances générales et n'exclut pas l'émergence de difficultés sectorielles, locales ou transitoires engendrées par l'intensification de la concurrence internationale. II indique que la globalisation des économies peut indéniablement être mise à profit pour favoriser la diffusion de meilleures conditions de travail dans les pays en développement.

\section{Conclusion}

Au stade actuel de l'évolution économique mondiale, on ne saurait affirmer que l'application de normes du travail moins rigoureuses dans certains pays en développement soit à l'origine des difficultés rencontrées par la main-d'oeuvre de faible qualification dans les pays où la réglementation sociale est plus sévère. L'avantage comparatif dont bénéficient certains pays en développement dans les secteurs où le niveau des salaires constitue une composante importante des coûts de production renvoie essentiellement aux paramètres du marché de l'emploi domestique et au niveau de productivité globale de leurs économies. De plus, l'intensification des échanges avec les pays à bas salaires n'explique que pour une faible partie la détérioration de la situation de la maind'oeuvre peu qualifiée dans les pays industrialisés. Cette catégorie subit avant tout les contrecoups des changements technologiques qui déplacent la demande de travail vers des profils plus qualifiés. Par ailleurs, l'ouverture aux échanges et aux investissements internationaux ne déclenche pas un phéno-

59 Cf. Bureau international du travail, L'emploi dans le monde. Un rapport du BIT, Genève, 1995, 83.

60 Cf. UNCTAD, World Investment Report 1994, New York and Geneva.

61 Cf. International Metalworkers' Federation (IMF), Trade and Workers' Rights. Time for a Link, Genève, 1988, 38-39. 
mène de dévaluation sociale généralisée. Dans les pays en développement, il apparaît au contraire que les performances à l'exportation sont souvent associées à une amélioration de la condition des travailleurs, en particulier en ce qui concerne leur rémunération. L'imposition de sanctions commerciales contre les pays qui n'appliquent pas certaines normes du travail pourrait donc aboutir à un résultat tout à fait paradoxal dans la mesure où ces derniers ne seraient plus en mesure de tirer profit des synergies positives entre la globalisation des économies et l'amélioration de la situation des travailleurs. En outre, les entraves ainsi posées aux échanges risqueraient de se retourner contre les pays exportateurs à normes élevées car il est fort probable que les marchés des pays pénalisés se fermeraient pour raison d'insolvabilité ou de représailles. L'option protectionniste se solderait immanquablement par un bilan négatif pour l'ensemble des partenaires commerciaux.

Les travaux entrepris par l'OIT sur la dimension sociale du commerce international n'ont pas permis, à ce stade, d'identifier les normes du travail qui doivent être respectées universellement. Les initiatives visant à renforcer les mécanismes de contrôle de l'OIT doivent toutefois être poursuivie. II y a également lieu de développer les mécanismes faisant appel à l'incitation tels que l'assistance technique et les codes de conduite à l'intention des entreprises transnationales.

II ressort de ce qui précède que les conditions posées plus haut d'une négociation, au sein de l'OMC, sur les interactions des normes du travail et du commerce ne sont pas réunies à ce jour. Si ce thème devait néanmoins figurer à l'ordre du jour de l'Organisation, il conviendrait de concevoir des solutions compatibles avec le caractère ouvert et équitable du système commercial multilatéral. L'éventuelle apparition de mesures commerciales liées aux normes du travail ne devrait pas servir de prétexte au protectionnisme. Dans cette perspective, il s'agirait d'écarter le modèle des moyens de défense commerciale qui, à l'instar des mesures antidumping, laissent une grande marge de discrétion aux Etats. On a en effet constaté que les réglementations antidumping sont trop facilement utilisées par les branches de production qui souhaitent éviter la concurrence des producteurs étrangers. ${ }^{62}$ Les mesures commerciales ne devraient intervenir qu'en dernier recours, lorsque tous les autres moyens d'action - en particulier les moyens incitatifs - auront été épuisés. De surcroît, elles devraient être soumises à un contrôle multilatéral rigoureux.

62 Cf. Horlick G. N., "How the GATT became Protectionist. An Analysis of the Uruguay Round Draft Final Antidumping Code", Journal of World Trade, 1993; Congressional Budget Office, How the GATT affects US Antidumping and Countervailing Duty Policy, The Congress of the United States, September 1994; Finger J. M., ed., Antidumping: How it works and Who get hurt, Ann Arbor, The University of Michigan Press, 1993. 